\title{
A Nomogram to Predict Acute Respiratory Distress Syndrome After Cardiac Surgery
}

\author{
Yan Liu, MD, ${ }^{1}$ Man Song, MD,${ }^{1}$ Lixue Huang, $M D,{ }^{2}$ Guangfa $Z$ hu, $M D, \mathrm{PhD}^{1,3}$ \\ ${ }^{1}$ Department of Infectious Diseases, Beijing Anzhen Hospital, Capital Medical University, Beijing Institute of Heart, Lung and Blood Vessel \\ Diseases, Beijing 100029, PR China; ${ }^{2}$ Department of Pulmonary and Critical Care Medicine, Center for Respiratory Diseases, China-Japan \\ Friendship Hospital, National Clinical Research, Center for Respiratory Diseases, 100029, PR China; ${ }^{3}$ Department of Pulmonary and \\ Critical Care Medicine, Beijing Anzhen Hospital, Capital Medical University, Beijing Institute of Heart, Lung and Blood Vessel Diseases, \\ Beijing 100029, PR China
}

\section{ABSTRACT}

Purpose: To establish a model to predict the risk of acute respiratory distress syndrome (ARDS) after cardiac surgery.

Methods: Data were collected on 132 ARDS patients, who received valvular or coronary artery bypass grafting surgery from January 2009 to December 2019. We developed the prediction model by multivariable logistic regression. Then, we used the coefficients for developing a nomogram that predicts ARDS occurrence. Internal validation was performed using resampling techniques to evaluate and optimize the model.

Results: All variables fit into the model, including albumin level before surgery (odds ratio [OR]: 0.96; 95\% confidence interval $[\mathrm{CI}]: 0.92,0.99 ; P=.01)$, cardiopulmonary bypass time (OR: $1.01 ; 95 \%$ CI: 1.00, 1.02; $P=.02$ ), APACHE II after surgery (OR: $1.21 ; 95 \% \mathrm{CI}: 1.13,1.29 ; P<.001)$, and history of diabetes (OR: 2.31; 95\% CI: 1.88, 3.87; $P<.001$ ); these were considered to build the nomogram. The score distinguished ARDS patients from non-ARDS patients with an AUC of 0.785 (95\% CI: $0.740,0.830)$ and was well calibrated (Hosmer-Lemeshow $P=.53$ ).

Conclusions: Our developed model predicted ARDS in patients undergoing cardiac surgery and may serve as a tool for identifying patients at high risk for ARDS after cardiac surgery.

\section{INTRODUCTION}

Acute respiratory distress syndrome (ARDS) seriously affects the prognosis of patients undergoing cardiac surgery, and it is associated with a mortality rate as high as $40 \%$ [Milot 2001; K. S. 2011; Kogan 2014]. Despite several years of research, effective treatments for ARDS still are relatively limited, and they include lung protective mechanical ventilation, fluid management, glucocorticoid administration, and other organ function maintenance measures [Forel 2012; Emir 2015; Grissom 2015]. Therefore, current research on

Received March 14, 2021; accepted March 29, 2021.

Correspondence: Dr: Guangfa Zhu, Department of Pulmonary and Critical Care Medicine, Beijing Anzhen Hospital, Capital Medical University, Beijing Institute of Heart, Lung and Blood Vessel Diseases, Beijing 100029, PR China; +86-1018910778607 (e-mail: guangfa_zbu@ccmu.edu.cn).
ARDS is focused on early detection and effective measures to prevent its occurrence and improve the prognosis of patients [Rubenfeld 2015]. The incidence and mortality of ARDS can be reduced by identifying the risk factors and establishing a lung injury prediction score (LIPS), allowing us to identify early high-risk patients with ARDS and take preventive measures before ARDS occurrence [Beitler 2014]. Thus far, there are only a few studies on the establishment of LIPS. The outcome of cardiac surgery significantly depends on the type of operation, operation process, blood transfusion during the operation, and postoperative factors, all of which can influence ARDS occurrence. To the best of our knowledge, no LIPS is specifically used to predict the occurrence of ARDS after cardiac surgery. Our study aimed at identifying high-risk ARDS patients early to assist clinicians in decision-making and take early preventive measures.

\section{METHODS}

Study population: Data from the department of cardiac surgery of our hospital were obtained for all patients who received valvular or coronary artery bypass grafting (CABG) surgery between 2009 and 2019. The research coordinators screened all patients for ARDS defined similarly to the Berlin Definition of ARDS [ARDS Definition Task Force 2012]. Chest radiography results were reviewed by two physicians. Any disagreement was resolved after discussion with a third physician for arbitration. All physicians underwent a consensus training session on the radiologic criteria for ARDS. All were blinded to the clinical status of the patients and the presence of other ARDS criteria. Data collected for each patient are shown in the next section. Patients with missing data were excluded from the analysis. Overall, 132 patients were included in the ARDS group. We selected all patients with the same sex and age who received valvular or CABG surgery in the same year to match each ARDS patient. We randomly selected five patients from all these candidates using a random number table in the same year. As a result, 660 patients were enrolled into the contrast group. Before enrolling into the study, approval of the local ethics committee was obtained (No. 20141103), and the study protocol was registered on www.ClinicalTrial.gov (ClinicalTrial.gov IDNCT02759770).

Measurement variables: Variables were chosen based on a previous literature review of risk factors for ARDS and 
Table 1. Baseline patients' characteristics and univariable analysis predicting ARDS

\begin{tabular}{|c|c|c|c|}
\hline & ARDS group & Contrast group & $P$ \\
\hline$N$ & 132 & 660 & \\
\hline Age (years) & $62.56 \pm 12.65$ & $61.02 \pm 11.74$ & .174 \\
\hline BMI & $24.21 \pm 3.67$ & $24.59 \pm 3.26$ & .233 \\
\hline HGB before, $g / L$ & $125.22 \pm 23.50$ & $133.73 \pm 21.00$ & $<.001$ \\
\hline ALB before, $g / L$ & $37.98 \pm 7.37$ & $40.70 \pm 6.22$ & $<.001$ \\
\hline EuroSCORE & $3.46 \pm 1.74$ & $2.65 \pm 1.70$ & .479 \\
\hline Operation time, hrs & $5.11 \pm 1.80$ & $4.20 \pm 1.09$ & $<.001$ \\
\hline CPB time, $\min$ & $99.50(0.00-170.00)$ & $0.00(0.00-84.25)$ & $<.001$ \\
\hline $\begin{array}{l}\text { Artery clamping time, } \\
\text { min }\end{array}$ & $57.50(0.00-106.75)$ & $\begin{array}{c}0.00 \\
(0.00-52.00)\end{array}$ & $<.001$ \\
\hline Blood loss, ml & $\begin{array}{c}800.00 \\
(500.00-1000.00)\end{array}$ & $\begin{array}{c}600.00 \\
(400.00-800.00)\end{array}$ & $<.001$ \\
\hline $\begin{array}{l}\text { Transfusion red blood } \\
\text { cell, ml }\end{array}$ & $\begin{array}{c}600.00 \\
(300.00-800.00)\end{array}$ & $\begin{array}{c}400.00 \\
(200.00-600.00)\end{array}$ & $<.001$ \\
\hline Transfusion plasma, ml & $0.00(0.00-400.00)$ & $0.00(0.00-0.00)$ & $<.001$ \\
\hline Transfusion platelet, $\mathrm{ml}$ & $0.00(0.00-0.00)$ & $0.00(0.00-0.00)$ & .448 \\
\hline APACHE II & $18.92 \pm 4.53$ & $16.17 \pm 3.00$ & $<.001$ \\
\hline Gender & - & - & .459 \\
\hline Male & $84(63.64 \%)$ & $442(66.97 \%)$ & \\
\hline Female & $48(36.36 \%)$ & $218(33.03 \%)$ & \\
\hline Smoking & - & - & .013 \\
\hline No & $82(62.12 \%)$ & $481(72.88 \%)$ & \\
\hline Yes & $50(37.88 \%)$ & $179(27.12 \%)$ & \\
\hline History of hypertension & - & - & .823 \\
\hline No & $72(54.55 \%)$ & $367(55.61 \%)$ & \\
\hline Yes & $60(45.45 \%)$ & $293(44.39 \%)$ & \\
\hline $\begin{array}{l}\text { History of cardiac } \\
\text { surgery }\end{array}$ & - & - & .055 \\
\hline No & $126(95.45 \%)$ & $648(98.18 \%)$ & \\
\hline Yes & $6(4.55 \%)$ & $12(1.82 \%)$ & \\
\hline History of diabetes & - & - & .002 \\
\hline No & $93(70.45 \%)$ & $543(82.27 \%)$ & \\
\hline Yes & $39(29.55 \%)$ & $117(17.73 \%)$ & \\
\hline History of COPD & - & - & $<.001$ \\
\hline No & 124 (93.94\%) & 651 (98.64\%) & \\
\hline Yes & $8(6.06 \%)$ & $9(1.36 \%)$ & \\
\hline History of AMI & - & - & .137 \\
\hline No & $91(68.94 \%)$ & $496(75.15 \%)$ & \\
\hline Yes & $41(31.06 \%)$ & $164(24.85 \%)$ & \\
\hline Operation position & - & - & $<.001$ \\
\hline Valve or CABG & $104(78.79 \%)$ & $633(95.91 \%)$ & \\
\hline Valve + CABG & $28(21.21 \%)$ & $27(4.09 \%)$ & \\
\hline
\end{tabular}

Table 2. Multivariable analysis predicting ARDS

\begin{tabular}{|c|c|c|c|}
\hline & Statistics & OR $(95 \% \mathrm{Cl})$ & $P$ \\
\hline HGB before, $g / L$ & $132.31 \pm 21.66$ & $0.99(0.98,1.00)$ & .1889 \\
\hline ALB before, $g / L$ & $40.25 \pm 6.50$ & $0.96(0.92,0.99)$ & .0082 \\
\hline Operation time, hours & $4.36 \pm 1.28$ & $1.05(0.83,1.31)$ & .6930 \\
\hline CPB time, $\min$ & $\begin{array}{c}0.00(0.00- \\
100.00)\end{array}$ & $1.01(1.00,1.02)$ & .0221 \\
\hline $\begin{array}{l}\text { Artery clamping time, } \\
\text { min }\end{array}$ & $0.00(0.00-62.00)$ & $0.99(0.98,1.01)$ & .3628 \\
\hline Blood loss, ml & $\begin{array}{c}600.00 \\
(400.00-862.50)\end{array}$ & $1.00(1.00,1.00)$ & .1378 \\
\hline $\begin{array}{l}\text { Transfusion red blood } \\
\text { cell, ml }\end{array}$ & $\begin{array}{c}400.00 \\
(200.00-600.00)\end{array}$ & $1.00(1.00,1.00)$ & .0003 \\
\hline Transfusion plasma, ml & $\begin{array}{c}0.00 \\
(0.00-200.00)\end{array}$ & $1.00(1.00,1.00)$ & .6032 \\
\hline APACHE II & $16.63 \pm 3.46$ & $1.21(1.13,1.29)$ & $<.0001$ \\
\hline Smoking & - & - & .1035 \\
\hline No & $563(71.09 \%)$ & 1.0 & \\
\hline Yes & $229(28.91 \%)$ & $1.51(0.92,2.48)$ & \\
\hline History of diabetes & - & - & .0015 \\
\hline No & $636(80.30 \%)$ & 1.0 & \\
\hline Yes & $156(19.70 \%)$ & $2.31(1.88,3.87)$ & \\
\hline History of COPD & - & - & .3372 \\
\hline No & 775 (97.85\%) & 1.0 & \\
\hline Yes & $17(2.15 \%)$ & $1.83(0.53,6.24)$ & \\
\hline Operation position & - & - & .5992 \\
\hline Valve or CABG & 737 (93.06\%) & 1.0 & \\
\hline Valve + CABG & $55(6.94 \%)$ & $1.51(0.65,3.48)$ & \\
\hline
\end{tabular}

additional risk factors for acute lung injury, due to other causes. Preoperative, operative, and postoperative variables were collected. Preoperative factors included age, sex, smoking status (whether the patient smoked during the recent one year or not), body mass index (BMI), history of acute myocardial infarction (AMI), cardiac surgery, hypertension, diabetes, chronic obstructive pulmonary disease (COPD), and levels of albumin (ALB) and hemoglobin (HGB) before surgery and EuroSCORE [Nashef 1999]. Operative factors included operation position, operation time, cardiopulmonary bypass (CPB) time, aortic clamping time, blood loss, and transfusion. Postoperative factors included the Acute Physiology and Chronic Health Evaluation II (APACHE II) score (calculated at the time of first day after surgery) and incubation time.

Statistical analysis: Categorical variables are reported as frequencies and proportions and continuous variables as mean (standard deviation) or median (interquartile range $[\mathrm{IQR}])$. Unadjusted associations between the covariates and primary outcome were evaluated using $\chi^{2}$ tests for categorical data, the $t$ test for normally distributed variables, and the 


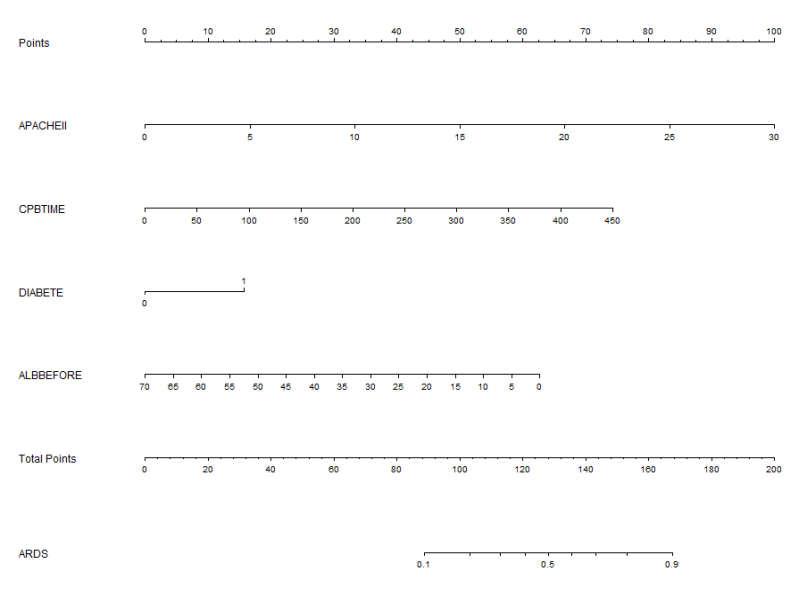

Figure 1. Nomogram for the prediction of acute respiratory distress syndrome (ARDS) after cardiac surgery, based on a multivariable model. Instructions: The patient's APACHE II points are located on the corresponding axis, and a straight line is drawn downward to the points axis to determine the number of points accumulated for the APACHE II score toward the probability of ARDS. This process is repeated for each variable, and the total number of points for all predictors is located on the points axis. A straight line is then drawn upward to determine the probability of ARDS in the patient.

Kruskal-Wallis test for nonparametric variables. Significant variables selected from the previous step were included in multivariable logistic regression and were then used to construct the model. Finally, we developed the prediction model using the entire data set. Internal validation of the model was performed using resampling (bootstrapping $=500$ ) techniques to evaluate the performance and optimize the developed model. To assess the discriminatory power of our model, the area under the receiver-operating characteristic curve (AUC) of the prediction scale was determined. The threshold score providing the best combination of sensitivity and specificity was determined by AUC analysis. Model calibration was assessed by using the Hosmer-Lemeshow test.

Statistical analyses were performed with the statistical software package R (http://www.R-project.org, The R foundation) and EmpowerStats (http://www.empowerstats.com, $\mathrm{X} \& Y$ solutions, Inc., Boston, MA, USA). A two-sided significance level of 0.05 was used to evaluate statistical significance.

\section{RESULTS}

Baseline characteristics of the patients and univariable analysis predicting ARDS: Descriptive characteristics for the overall population are listed in Table 1. HGB and ALB levels before surgery in the ARDS group were lower than those in the contrast group. Operation time, CPB time, and artery clamping time were longer in the ARDS group than the contrast group. Both the amount of blood loss and transfusion red blood cell and plasma were much higher in the ARDS group than the contrast group. APACHE II score after surgery in

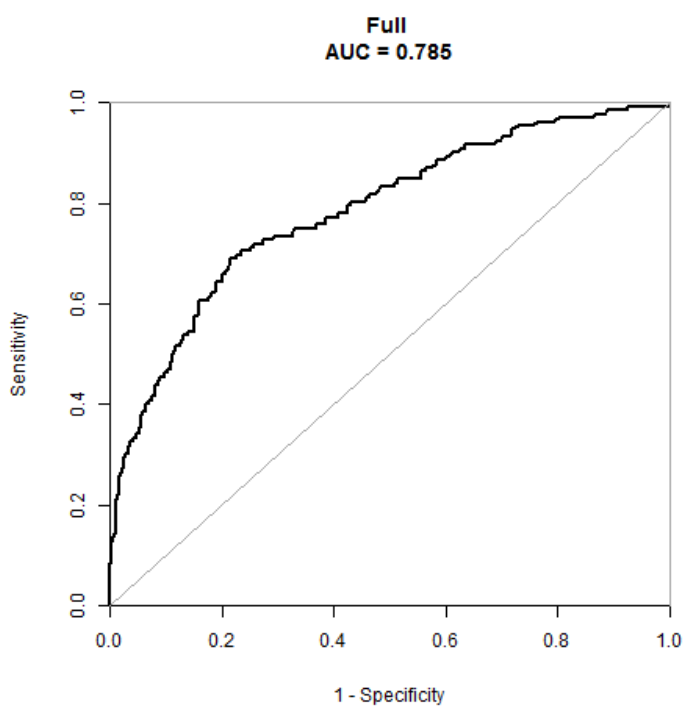

Figure 2. Receiver operating characteristic curves for acute respiratory distress syndrome (ARDS) development.

the ARDS group was higher than that in the contrast group. There were more smokers in the ARDS group than the contrast group, and more ARDS patients had a history of diabetes and COPD. More patients in the ARDS group (21\%) had a combined surgery than the contrast group (4\%).

Multi-variable analysis predicting ARDS: The role of HGB, ALB, operation time, CPB time, artery clamping time, blood loss, transfusion red blood cell, transfusion plasma, APACHE II score, smoking status, history of diabetes and COPD, and operation position were subjected to multivariable analysis (Table 2). Covariates were then excluded, according to their statistical significance. Eventually, ALB level before surgery, history of diabetes, CPB time, and APACHE II score were the candidate variables. We used the most parsimonious model based on the statistically significant effect of the variables included.

Sensitivity analyses: Coefficients from the multivariable analysis were utilized to build a nomogram for predicting significant ARDS (Figure 1). Discrimination of the ARDS score was evaluated by analysis of the AUC, which was found to be 0.785 (95\% confidence interval: 0.76-0.84) (Figure 2). The prediction model also was well calibrated (HosmerLemeshow $P=.53$ ).

\section{DISCUSSION}

ARDS is a form of noncardiogenic pulmonary edema, due to alveolar injury secondary to inflammation, that is manifested clinically by the acute onset of bilateral infiltrates (visible on chest radiograph) and arterial hypoxemia [ARDS Definition Task Force 2012; Sweeney 2016]. Aspiration, sepsis, trauma, shock, and infection are common causes of ARDS. Since cardiac surgery also is associated with these phenomena, there is also a high risk of ARDS after cardiac surgery. Indeed so, ARDS has been reported in $0.4-2.5 \%$ 
cases after cardiac surgery, and it is associated with a mortality of 15-68.4\% [Milot 2001; Michalopoulos 2006; Kogan 2014]. Postoperative ARDS is associated with an increase in in-hospital mortality and duration of stay in intensive care and hospital [Dowdy 2006; Wilcox 2010].

Several studies have been conducted to identify the risk factors of ARDS after its definition in 1973. These include previous cardiac surgery [Milot 2001; Kogan 2014], diabetes mellitus [Kor 2011; Tamayo 2012], COPD [Kaul 1998; Kor 2011], gastroesophageal reflux disease [Kor 2011], recent cigarette smoking [Kogan 2014], complex cardiac surgery [Kogan 2014] and transfusion [Vlaar 2011; Kogan 2014], blood loss [Tamayo 2012] during surgery, and increased CPB time [Christenson 1996; Tamayo 2012]. We could collect information on all these factors in our study except gastroesophageal reflux disease, since there were only four $(0.51 \%)$ patients admitted, according to their medical records. Moreover, we also found evidence of preoperative nutritional status, including ALB and HGB levels, as risk factors from our data collection. Since EuroSCORE is one of the most widely used systems for assessing early mortality in cardiac surgical patients, it was included into our variables. Among the many intensive care unit (ICU) scoring systems, APACHE II is the most commonly used severity-of-disease scoring system in ICUs worldwide [Salluh 2014]. We added APACHE II score to our prediction model to evaluate the patient's state after surgery.

To the best of our knowledge, several prediction models have been created for the risk of ARDS/acute lung injury [Wilcox 2010; Trillo-Alvarez 2011; Elie-Turenne 2012]. Alvarez et al performed research on adult ICU patients, and their model was externally validated using another cohort population (AUC 0.840). A second study included patients admitted to the emergency department, similar to the previous study, and the model used in this study was used to evaluate the risk of ARDS (AUC 0.797). Kor et al carried out a study that included patients who underwent cardiac surgery and other high-risk operations, including vascular surgery and thoracic surgery, and their prediction model outperformed the original score (AUC 0.840). Nevertheless, operative and postoperative factors were not analyzed. CABG and valve surgery are the most common procedures performed for cardiac surgery. These two types of surgery were included in our study to reduce patient selection bias. To the best of our knowledge, ours is the first nomogram to evaluate the risk of ARDS after cardiac surgery and is more concise than the above-mentioned ones. Our model included four variables: diabetes, ALB level before surgery, CPB time, and APACHE II score. Thus, our model evaluated the development of ARDS considering preoperative, intraoperative, and postoperative factors with the AUC as 0.785 ; it also was well calibrated (Hosmer-Lemeshow $P=.53$ ).

Previous retrospective studies have shown conflicting results, regarding ARDS development in patients with diabetes. Some studies showed that patients with diabetes had a decreased incidence of ARDS [Moss 2000; Gong 2005; Honiden 2009]. Diabetes may have a potential immunomodulatory effect. However, insulin therapy in diabetes patients could also act as a confounding factor because the protective effect seen in diabetes patients could also be because of the insulin therapy those patients were receiving [Honiden 2009]. Preclinical studies have demonstrated the protective benefits of insulin therapy in lung injury secondary to trauma [Donnelly 2007] and when used to maintain euglycemia in ARDS secondary to endotoxemia [Chen 2006]. In contrast, some studies, including ours, reported that diabetes is associated with the development of ARDS [Tamayo 2012; Kor 2011]. Boyle et al. [Boyle 2018] carried out a global, multicenter, prospective, observational study to clarify the association between diabetes and ARDS recently. Their results showed no association between diabetes and outcomes of ARDS. Their study included inhalational injury, pulmonary contusion, pulmonary vasculitis, and drowning, alongside gastric aspiration and pneumonia, as direct risk factors. Surgical factors, especially cardiac surgery, were not included, which may explain the difference in results to some extent between their study and ours. The association between diabetes and ARDS among cardiac surgery patients needs to be confirmed by larger-scale multicenter studies and basic research.

Low ALB level may indicate a poor nutritional status of patients. Hypoproteinemia also affects the patient's colloidal osmotic pressure, promotes pulmonary edema, and affects the patient's oxygenation capacity. Moreover, a study showed that low ALB level is a predictor for ARDS after abdominal surgery [Bingzheng 2019]. Our research confirmed this result.

Increased CPB time during surgery was a risk factor for ARDS, which was confirmed in another study [Tamayo 2012]. Two hypotheses can be proposed regarding the mechanism of $\mathrm{CPB}$ causing ARDS. One is ischemia of the lung during CPB. Ischemia-reperfusion of the lungs stimulates an inflammatory response, thereby damaging the lungs [Schlensak 2001; Kogan 2014]. The other hypothesis is related to systemic inflammatory response syndrome (SIRS) [Davies 1997]. When blood contacts the extracorporeal circulation device and protamine neutralizes heparin, they activate the SIRS pathway [Warltier 2002], which leads to the swelling of lung epithelial cells, leaking of protein into the lung interstitium, and increase in hydrolase level, which eventually leads to alveolar consolidation [Edmunds 1995].

The limitation of our study is the use of internal validation, which was performed using resampling techniques to evaluate the performance of and to optimize the developed model. Internal validation has less power than external validation using a prospective population. As a result, additional prospective studies are required to further evaluate our model.

\section{CONCLUSIONS}

We used a nomogram to predict the risk of ARDS among cardiac surgery patients, including diabetes, ALB level before surgery, CPB time, and APACHE II score. We can use the above variables of cardiac surgery patients to evaluate their risk of ARDS, and then we can take preventive measures early and pay more attention to patients with these risk factors. Our prediction model is more concise than other methods, without any loss in predictive power, since other models needed 
to separately calculate the score, according to the variable and then obtain the total score to evaluate the risk of ARDS. However, our prediction model simply uses a nomogram, which is more convenient and accurate.

\section{ACKNOWLEDGEMENT}

This work was granted by the Capital Health Research and Development of Special (grant.no. SF2016-2-1052) and the National Natural Science Foundation of China (grant. no. 81970067).

Thanks to Vinay $S$ for the language support.

\section{REFERENCES}

ARDS Definition Task Force, Ranieri VM, Rubenfeld GD, Thompson BT, Ferguson ND, Caldwell E, et al. 2012. Acute respiratory distress syndrome: the Berlin Definition. JAMA. 307(23):2526-2533.

Beitler Jeremy R, Schoenfeld David A, Taylor TB. 2014. Preventing ARDS: progress, promise, and pitfalls. Chest. 146(4).

Bingzheng X, Yangli G, Yangzhen L, Qianqian C, Hongjin Z. 2019. Risk factors and prognosis of acute respiratory distress syndrome following abdominal surgery. Exp Ther Med. 17(1).

Boyle AJ, Madotto F, Laffey JG, Bellani G, Pham T, Pesenti A, et al. 2018. Identifying associations between diabetes and acute respiratory distress syndrome in patients with acute hypoxemic respiratory failure: an analysis of the LUNG SAFE database. 22(1).

Chen HI, Yeh DY, Liou H, Kao S. 2006. Insulin attenuates endotoxininduced acute lung injury in conscious rats. Critical Care Medicine. 34(3).

Christenson JT, Aeberhard JM, Badel P, Pepcak F, Maurice J, Simonet F, et al. 1996. Adult respiratory distress syndrome after cardiac surgery. Cardiovasc Surg. 4(1):15-21.

Davies MG, Hagen PO. 1997. Systemic inflammatory response syndrome. Br J Surg. 84(7).

Donnelly M, Condron C, Murray P, Bouchier-Hayes D. 2007. Modulation of the Glycemic Response Using Insulin Attenuates the Pulmonary Response in an Animal Trauma Model. The Journal of Trauma: Injury, Infection, and Critical Care. 63(2).

Dowdy DW, Eid MP, Dennison CR, Mendez-Tellez PA, Herridge MS, Guallar E, et al. 2006. Quality of life after acute respiratory distress syndrome: a meta-analysis. Intensive Care Med. 32(8):1115-1124.

Edmunds LH. 1995. Why cardiopulmonary bypass makes patients sick: strategies to control the blood-synthetic surface interface. Advances in cardiac surgery. 6.

Elie-Turenne MC, Hou PC, Mitani A, Barry JM, Kao EY, Cohen JE, et al. 2012. Lung injury prediction score for the emergency department: first step towards prevention in patients at risk. Int J Emerg Med. 5(1):33.

Emir F, Kor DJ, Ognjen G. 2015. Prevention of acute respiratory distress syndrome. Curr Opin Crit Care. 21(1).

Forel J, Voillet F, Pulina D, Gacouin A, Perrin G, Barrau K, et al. 2012. Ventilator-associated pneumonia and ICU mortality in severe ARDS patients ventilated according to a lung-protective strategy. 16(2).

Gong MN, Thompson BT, Williams P, Pothier L, Boyce PD, Christiani DC. 2005. Clinical predictors of and mortality in acute respiratory distress syndrome: Potential role of red cell transfusion. Critical Care Medicine. 33(6).

Grissom CK, Hirshberg EL, Dickerson JB, Brown SM, Lanspa MJ, Liu $\mathrm{KD}$, et al. 2015. Fluid management with a simplified conservative protocol for the acute respiratory distress syndrome*. Critical care medicine. $43(2)$.

Honiden S, Gong MN. 2009. Diabetes, insulin, and development of acute lung injury. Critical Care Medicine. 37(8).

K. S. B, R. S, H. P, A. W, V. V. 2011. Mortality risk prediction in cardiac surgery: comparing a novel model with the EuroSCORE. Acta Anaesthesiol Scand. 55(3).

Kaul TK, Fields BL, Riggins LS, Wyatt DA, Jones CR, Nagle D. 1998. Adult respiratory distress syndrome following cardiopulmonary bypass: incidence, prophylaxis and management. J Cardiovasc Surg (Torino). 39(6).

Kogan A, Preisman S, Levin S, Raanani E, Sternik L. 2014. Adult respiratory distress syndrome following cardiac surgery. J Card Surg. 29(1):41-46.

Kor DJ, Warner DO, Alsara A, Fernández-Pérez ER, Malinchoc M, Kashyap R, et al. 2011. Derivation and Diagnostic Accuracy of the Surgical Lung Injury Prediction Model. Anesthesiology. 115(1):117-128.

Michalopoulos A, Prapas S, Falagas ME. 2006. The incidence of adult respiratory distress syndrome in patients undergoing off-pump coronary artery bypass grafting surgery. Eur J Anaesthesiol. 23(1):80.

Milot J, Perron J, Lacasse Y, Létourneau L, Cartier PC, Maltais F. 2001. Incidence and predictors of ARDS after cardiac surgery. Chest. 119(3):884-888.

Moss M, Guidot DM, Steinberg KP, Duhon GF, Treece P, Wolken R, et al. 2000. Diabetic patients have a decreased incidence of acute respiratory distress syndrome. Critical Care Medicine. 28(7).

Nashef SA, Roques F, Michel P, Gauducheau E, Lemeshow S, Salamon R. 1999. European system for cardiac operative risk evaluation (EuroSCORE). Eur J Cardiothorac Surg. 16(1):9-13.

Rubenfeld GD. 2015. Who cares about preventing acute respiratory distress syndrome. Am J Respir Crit Care Med. 191(3):255-260.

Salluh JI, Soares M. 2014. ICU severity of illness scores: APACHE, SAPS and MPM. Curr Opin Crit Care. 20(5):557-565.

Schlensak C, Doenst T, Preußer S, Wunderlich M, Kleinschmidt M, Beyersdorf F. 2001. Bronchial artery perfusion during cardiopulmonary bypass does not prevent ischemia of the lung in piglets: assessment of bronchial artery blood flow with fluorescent microspheres. Eur J Cardiothorac Surg. 19(3).

Sweeney RM, McAuley DF. 2016. Acute respiratory distress syndrome. Lancet. 388(10058):2416-2430.

Tamayo E, Álvarez FJ, Martínez-Rafael B, Bustamante J, Bermejo-Martin JF, Fierro I, et al. 2012. Ventilator-associated pneumonia is an important risk factor for mortality after major cardiac surgery. J Crit Care. 27(1):18-25.

Trillo-Alvarez C, Cartin-Ceba R, Kor DJ, Kojicic M, Kashyap R, Thakur $\mathrm{S}$, et al. 2011. Acute lung injury prediction score: derivation and validation in a population-based sample. Eur Respir J. 37(3):604-609.

Vlaar AP, Hofstra JJ, Determann RM, Veelo DP, Paulus F, Kulik W, et al. 2011. The incidence, risk factors, and outcome of transfusion-related acute lung injury in a cohort of cardiac surgery patients: a prospective nested case-control study. Blood. 117(16):4218-4225. 
Warltier DC, Laffey JG, Boylan JF, Cheng DCH. 2002. The Systemic Inflammatory Response to Cardiac Surgery: Implications for the Anesthesiologist. Anesthesiology. 97(1).
Wilcox M, Herridge M. 2010. Long-Term Outcomes in Patients Surviving Acute Respiratory Distress Syndrome. Semin Respir Crit Care Med. $31(1)$. 